\title{
REFERENCIA AL ARTÍCULO "ANÁLISIS MULTIVARIADO DE RECIDIVA Y PROGRESIÓN EN EL CARCINOMA DE CÉLULAS TRANSICIONALES DE VEJIGA EN ESTADIO T1. VALOR PRONÓSTICO DE p53 Y ki67"
}

\author{
J. PALOU REDORTA, F. MILLÁN RODRÍGUEZ
}

Servicio de Urología. Fundació Puigvert. Barcelona.

Actas Urol Esp. 27 (6): 478, 2003

$\mathrm{E}$ 1 motivo de esta carta es para aportar algunos comentarios sobre el artículo "Análisis multivariado de recidiva y progresión en el carcinoma de células transicionales de vejiga en estadio T1. Valor pronóstico de p53 y ki67" publicado en la Revista Actas Urológicas Españolas 27 (2): 132-141, 2003.

Mencionar que se trata de un estudio con un buen número de pacientes con un análisis pormenorizado de bastantes variables y con la inclusión en un análisis multivariante de tumor vesical superficial T1 del estudio de p53 y ki67.

Asimismo se comenta que se analizaron 177 variables para el estudio; suponemos que se incluyeron 177 variables por paciente en la base de datos y luego se analizaron sólo los más relevantes, ya que un estudio multivariante con 175 pacientes y 177 variables analizadas, sólo por azar puede concluir un gran número de variables con significación estadística.

En el mismo apartado se comenta que "todos los pacientes recibieron algún tipo de tratamiento quimio o inmunoterápico intravesical tras la RTU del tumor primario". No se refiere las indicaciones, grupos de tratamiento y no se ha analizado la repercusión de dicho tratamiento sobre la evolución y resultados obtenidos. Es conocida la influencia de la quimioterapia, y más de la inmunoterapia, en la recurrencia $^{1}$ y también en la progresión, sobre todo, en las terapias de mantenimiento ${ }^{2}$. Si en esta serie de pacientes estadio T1, los tumores de G3 o G2 múltiples recibieron inmunoterapia, se habrá obtenido un cambio en la evolución natural de dichos tumores, y puede ser la explicación de menor recurrencia de los G2 y G3 (RR=0,81 y 0,69) respecto a G1.

Dado que el patrón de crecimiento sólido de los tumores va a menudo asociado a tumores de alto grado, es lógico pensar que puede tratarse de un factor pronóstico en el análisis multivariante, aunque es curioso que la progresión no se relacione con el grado ni con el aspecto macroscópico. En varios estudios publicados, el grado normalmente se comporta, en todos ellos, como factor pronóstico en la progresión ${ }^{1}$.

Por último, otro factor pronóstico asociado a la progresión es la recurrencia temprana (en los primeros tres meses) ${ }^{3,4}$; la inclusión de este factor podría también influir en el resultado y aportar mayor información al plantear factores pronósticos en el estadio T1. Aunque esta aportación se ha estudiado sobretodo en tumores de alto grado y en el estudio presentado sólo representa un 19,4\%.

\section{REFERENCIAS}

1. MILLÁN RODRÍGUEZ F, CHÉCHILE-TONIOLO G, SALVADOR-BAYARRI J, PALOU J, VICENTE-RODRÍGUEZ J.: Multivariate analysis of the prognostic factors of primary superficial bladder cancer. J Urol 2000; 163: 73-78.

2. SILVESTER RJ, VAN DER MEIJDEN AP, LAMM DL: Intravesical bacillus Calmette-Guerin reduces the risk of progresion in patients with superficial bladder cancer: a meta-analysis of the published results of randomized clinical trials. J Urol 2002; 168: 1964-1970.

3. PALOU J, ROSALES A, MILLÁN F, ZARAGOZA R, SALVADOR J, VICENTE J.: Clinical prognostic factors of recurrence and progresion in TCC stage T1 G3 treated with BCG. BJU International 2000; 86 (supl. 3): 3.

4. SOLSONA E, IBORRA I, DUMONT R, RUBIO-BRIONES J, CASANOVA J, ALMENAR S.: The 3-month clinical response to intravesical therapy as a predictive factor for progression in patients with high risk superficial bladder cancer. J Urol 2000; 164: 685-689.

\section{Dr. J. Palou}

Servicio de Urología.

Fundació Puigvert

C/ Cartagena, 340

08025 Barcelona

(Trabajo recibido el 24 abril de 2003) 